\title{
Glucocorticoid use in patients with adrenal insufficiency following administration of the COVID-19 vaccine: a pituitary society statement
}

\author{
Laurence Katznelson ${ }^{1}[$ ] $\cdot$ Mônica Gadelha²
}

Accepted: 19 January 2021 / Published online: 10 February 2021

(c) The Author(s), under exclusive licence to Springer Science+Business Media, LLC part of Springer Nature 2021

\begin{abstract}
Purpose Side effects of the coronavirus disease 2019 (COVID-19) vaccines include pain at the injection site, fatigue, headache, myalgias, arthralgias, chills, and fever, all of which can be early indicators of an increased need for glucocorticoid replacement in patients with adrenal insufficiency. The Pituitary Society surveyed its membership to understand planned approaches to glucocorticoid management in patients with adrenal insufficiency who will receive a COVID-19 vaccine. Methods Members were asked to complete up to 3 questions regarding their planned approach for use of glucocorticoid replacement in patients with proven adrenal insufficiency.

Results Surveys were sent to 273 members and 103 responded. Thirty-six percent plan to recommend that patients automatically increase glucocorticoid dosage with administration of the first vaccine injection. Of these, $84 \%$ plan to increase glucocorticoid dose on the day of vaccination, and $49 \%$ plan to increase glucocorticoid dose prior to vaccination. Of the $64 \%$ who do not plan to recommend automatic glucocorticoid dose increase with vaccine administration, $88 \%$ plan to increase the dose if the patient develops a fever, and $47 \%$ plan to increase the dose if myalgias and arthralgias occur.

Conclusions Most clinicians plan to maintain the current glucocorticoid dose with vaccine administration. The vast majority plan and to increase glucocorticoid dose in case of fever, and just under half in case of arthralgias and myalgias. These survey results offer suggested management guidance for glucocorticoid management in patients with adrenal insufficiency.
\end{abstract}

Keywords Adrenal insufficiency · COVID-19 · Vaccines · Glucocorticoid replacement

Availability of vaccines is a giant step in combating the coronavirus disease 2019 (COVID-19) pandemic. Side effects of the Pfizer-BioNTech and Moderna vaccines include up to several days of pain at the injection site, fatigue, headache, myalgias, arthralgias, chills, and fever [1,2]. These signs and symptoms can be early indicators of an increased need for glucocorticoid replacement in patients with adrenal insufficiency $[3,4]$. Other than a case report of adrenal crisis in a patient with Addison's disease following routine

Laurence Katznelson

lkatznelson@stanford.edu

1 Departments of Medicine and Neurosurgery, Stanford University, 875 Blake Wilbur Dr., MC 5821, Stanford, CA 94305-5821, USA

2 Neuroendocrinology Research Center/Endocrinology Section, Medical School and Hospital Universitário Clementino Fraga Filho, Universidade Federal do Rio de Janeiro, Rio de Janeiro, Brazil administration of influenza, Tdap, and pneumococcal vaccines [5], there is no information on routine management of primary or secondary adrenal insufficiency following administration of these vaccines, and we do not yet know the potential impact of glucocorticoids on immune response to the vaccine.

The Pituitary Society surveyed its membership to understand planned approaches to glucocorticoid management in patients with adrenal insufficiency who will receive a COVID-19 vaccine with a goal of using survey results to inform the larger community of suggested best practices.

Surveys were developed using SurveyMonkey (San Mateo, CA) with branch logic. Members were asked to complete up to 3 questions regarding their planned approach for use of glucocorticoid replacement in patients with proven adrenal insufficiency (Table 1). The survey was closed after 3 days and results were collected and analyzed.

Surveys were sent to 273 members and 103 responded (38\% response rate). As shown in Fig. 1, 36\% (37/103) plan 
Table 1 Survey questions

Question 1

Question 2

Question 3

Question 4

Question 5
Following administration of the COVID-19 vaccine in a patient with proven adrenal insufficiency, do you plan to recommend that your patient automatically increase the glucocorticoid dosage?

If yes, answer questions $2 \& 3$

If no, answer questions $4 \& 5$

If the answer to question 1 was YES, will you recommend raising the glucocorticoid dose prior to the vaccination?

If yes, go to question 3

If no, go to question 3

If the answer to question 1 was YES, will you recommend raising the dose on the day of the vaccination?

If yes, end questionnaire

If no, end questionnaire

If the answer to question 1 was $\mathrm{NO}$, will you recommend that the patient increase the glucocorticoid dose for fever?

If yes, go to question 5

If no, go to question 5

If the answer to question 1 was NO, will you recommend that the patient increase the glucocorticoid dose for muscle and joint pains?

If yes, end questionnaire

If no, end questionnaire to recommend that patients automatically increase their glucocorticoid dosage with administration of the first vaccine injection. Of these, $84 \%$ (31/37) plan to increase glucocorticoid dose on the day of vaccination, and 49\% (18/37) plan to increase glucocorticoid dose prior to vaccination.

By contrast, 64\% (66/103) do not plan to recommend an automatic glucocorticoid dose increase with vaccine administration. Of these, $88 \%(58 / 66)$ plan to increase the dose if the patient develops a fever following administration, and $47 \%(31 / 66)$ plan to increase the dose if myalgias and arthralgias occur.

Thus, most clinicians plan to maintain the current glucocorticoid dose with vaccine administration. The vast majority of these clinicians plan to increase glucocorticoid dose with fever, and just under half plan to increase the dose with associated arthralgias and myalgias, known vaccine side effects.

These survey results offer a glimpse into the planned approach of our members for glucocorticoid management in patients with adrenal insufficiency. This survey does not reflect results of a trial on efficacy of glucocorticoid management in patients receiving the vaccine nor the impact of a particular glucocorticoid dose on the vaccine immune response. Importantly, these results do offer suggested management guidance based on responses from experienced clinicians treating pituitary diseases.

Fig. 1 Survey responses

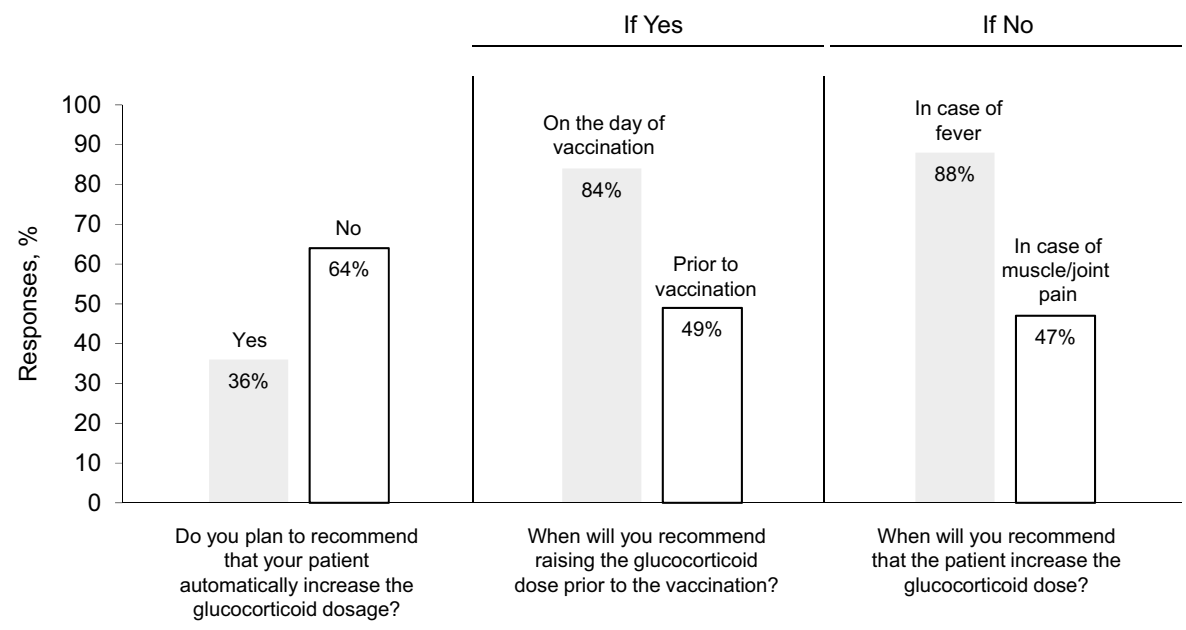


Acknowledgements The authors thank Dr. A.J. van der Lely, President, and Bari Laner, Director, of the Pituitary Society for assistance with this project.

Funding Development of this manuscript was supported by the Pituitary Society, for which Dr. Gadelha is President-Elect. The authors did not receive support from any organization for the submitted work.

\section{Compliance with ethical standards}

Conflict of interest The authors have no relevant financial or non-financial interests to disclose.

\section{References}

1. Full Emergency Use Authorization (EUA) Prescribing Information: Pfizer-BioNtech COVID-19 Vaccine. https://www.cvdva ccine-us.com/images/pdf/fact-sheet-for-hcp-administering-vacci ne-vaccination-providers-full-eua-prescribing-infor matio n.pdf\#page $=11$ (2020). Accessed 21 Dec 2020

2. Full Emergency Use Authorization (EUA) Prescribing Information: moderna COVID-19 Vaccine. https://www.modernatx.com/ covid19vaccine-eua/eua-fact-sheet-providers.pdf\#page $=8$ (2020). Accessed 21 Dec 2020

3. Bornstein SR, Allolio B, Arlt W et al (2016) Diagnosis and treatment of primary adrenal insufficiency: an Endocrine Society clinical practice guideline. J Clin Endocrinol Metab 101:364-389

4. Fleseriu M, Hashim IA, Karavitaki N et al (2016) Hormonal replacement in hypopituitarism in adults: an Endocrine Society clinical practice guideline. J Clin Endocrinol Metab 101:3888-3921

5. Major A, Chacko K (2018) Routine vaccinations as a precipitant of adrenal crisis in adrenal insufficiency. Intern Med J 48:360-361

Publisher's Note Springer Nature remains neutral with regard to jurisdictional claims in published maps and institutional affiliations. 